\title{
Entre recadrage et innovation : les modélisations de la culture humaniste sur le web
}

\section{François Quet}

\section{(2) OpenEdition \\ 1 Journals}

Édition électronique

URL : http://journals.openedition.org/trema/3326

DOI : $10.4000 /$ trema.3326

ISSN : 2107-0997

\section{Éditeur}

Faculté d'Éducation de l'université de Montpellier

\section{Édition imprimée}

Date de publication : 1 mai 2015

Pagination : $78-91$

ISSN : 1167-315X

\section{Référence électronique}

François Quet, «Entre recadrage et innovation : les modélisations de la culture humaniste sur le web », Tréma [En ligne], 43 | 2015, mis en ligne le 25 juin 2015, consulté le 19 avril 2019. URL : http:// journals.openedition.org/trema/3326 ; DOI : 10.4000/trema.3326

Ce document a été généré automatiquement le 19 avril 2019.

Trema 


\title{
Entre recadrage et innovation : les modélisations de la culture humaniste sur le web
}

\author{
François Quet
}

1 L'introduction de la culture humaniste à l'école a déjà donné lieu à une importante production théorique, critique ou pédagogique. Si l'on admet que celle de l'histoire des arts lui est étroitement corrélée, la liste est longue des contributions, articles et ouvrages qui se situent en amont, accompagnent la présentation des premiers textes officiels et conduisent à l'émergence d'exigences nouvelles pour le système éducatif, ou qui s'efforcent en aval de traduire de façon empirique ce que les acteurs institutionnels entendent faire de ces nouvelles prescriptions.

2 Les sites académiques ou d'inspections départementales déclinent des propositions ou des exemples d'actions pédagogiques qui illustrent les mises en œuvre de l'enseignement de la culture humaniste dans le premier degré, mais qui de par leur sélection même sur des espaces institutionnels, modélisent les actions à venir. Ressources incomparables, ces séquences développées ou ces projets innovants sont donc intéressants comme traductions/interprétations des programmes mais aussi comme instruments de formation: références légitimées par les inspections au service par exemple de l'élaboration de nouvelles séquences et de nouveaux projets. S'il ne s'agit pas à proprement parler de documents de formation, il s'agit presque toujours de travaux conduits en collaboration avec des conseillers pédagogiques, et de toutes façons, sanctionnés par une publication qui leur donne valeur d'exemple à suivre.

3 Après avoir posé quelques éléments de réflexion pour cerner ce qui, à mon avis, est problématique dans l'invocation de la culture humaniste à l'école, - étape au cours de laquelle je serai conduit à évoquer les espoirs qu'on lui fait porter, les difficultés relatives à l'imprécision de sa définition et les difficultés de sa mise en œuvre -, je voudrais montrer comment à un instant " $t$ " de l'institution, les acteurs s'emparent d'une commande institutionnelle, la modifient, la transposent, la réinventent. 


\section{Les doigts glacés}

4 Je dois à la lecture de Jacques Bouveresse ${ }^{1}$ la découverte d'un roman peu connu de Dickens. Dans Temps difficiles ${ }^{2}$, l'auteur d'Oliver Twist, met en relation le développement industriel d'une ville du centre de l'Angleterre et la mise en œuvre d'une éducation exclusivement basée sur «les faits» et qui s'interdit jusqu'à l'usage du mot «Imagination ». Mais le caractère délirant du personnage principal, directeur de l'école un peu spéciale de cette cité à demi fictive (où les élèves sont des numéros ${ }^{3}$ et où le cheval ne se définit que par l'addition de connaissances vérifiables : "Quadrupède. Herbivore. Quarante dents, etc. »), permet de souligner la dérive de la formation des enseignants que le romancier souhaite dénoncer.

5 Voici par exemple le portrait du professeur nouvellement recruté par le personnage central de Temps difficiles :

«M. M'Choakkumchild commença dans son meilleur style. Lui et quelques cent quarante autres maitres d'école avaient été récemment façonnés en même temps dans la même fabrique, selon les mêmes principes, comme autant de pieds de pianos à queue. On l'avait fait passer par une multitude infinie d'épreuves et il avait dû répondre à des montagnes de questions qui toutes étaient des casse-têtes. Il savait sur le bout des doigts, de ses dix doigts glacés, l'orthographe, l'étymologie, la syntaxe, la prosodie, la biographie, l'astronomie, la géographie, la cosmographie générale, les sciences à nombres complexes, l'algèbre, l'arpentage et le nivellement des sols, la musique vocale et le dessin d'après modèle. Il s'était durement frayé un chemin jusqu'au programme $B$ du très honorable Conseil Privé de sa Majesté et il avait réussi à enlever tout intérêt aux plus hautes branches des mathématiques et des sciences physiques, aux langues française, allemande, latine et grecque. Il savait tout ce qui avait rapport aux bassins hydrographiques du monde entier (quels qu'ils fussent), il connaissait l'histoire de tous les peuples et de toutes les montagnes, toutes les productions, les mœurs et coutumes de tous les pays, et quelles en étaient les frontières et la position sur les trente-deux divisions du compas. Un peu trop savant Mr. M'Choakkumchild ; si seulement il en avait appris un peu moins, comme il aurait été capable d'en enseigner davantage ${ }^{4}$. »

On commencera par noter les différences : nul doute que l'encyclopédisme sur lequel se fonde la formation des maitres de l'Angleterre victorienne est assez éloigné de ce qui se pratique aujourd'hui. Encore que... à y regarder de plus près, si la palette des savoirs requis s'est singulièrement réduite par rapport à l'image satirique qu'en donne Dickens, il y aurait probablement beaucoup à dire sur la nature des savoirs aujourd'hui impliqués dans la formation (et sur la prolifération de métaconnaissances didactiques ou pédagogiques par exemple). Quoiqu'il en soit, ce plaidoyer pour une formation moins savante, et plus humaine fait écho à nombre de préoccupations véritablement contemporaines : le discours critique de Dickens sert d'ailleurs de point de départ à la réflexion de Martha Nussbaum ${ }^{5}$. Les doigts glacés de l'enseignant révèlent le cœur froid que masque un vain savoir et la tête bien pleine, dont personne ne prendra le temps de s'assurer qu'elle est aussi bien faite. Le roman de Dickens invite à s'interroger sur les conséquences d'un enseignement qui se défie de la créativité et de l'imagination jusque dans la formation des maitres. 


\section{La place de l'émotion}

7 L'appel à la culture humaniste répond au désir ambigu de former le citoyen du XXIe siècle en privilégiant les émotions démocratiques ${ }^{6}$. Les premières pages du livre de Martha Nussbaum décrivent une crise, américaine certes, mais que de nombreux critiques et polémistes ont cru déceler dans le contexte éducatif français: désaffection pour les humanités, baisse des connaissances culturelles, statut toujours plus incertain des enseignements artistiques. On ne reviendra pas sur un constat alarmiste auquel donnerait sans doute raison la toute récente élaboration à la hâte de maquettes de Master d'enseignement, entièrement orientées par un concours de recrutement qui n'accorde aux humanités qu'une place très réduite. L'art et les artistes ne sont pas « les serviteurs fidèles de quelque idéologie que ce soit ", c'est la raison pour laquelle ils sont considérés comme des ennemis par tous ceux qui souhaitent former des citoyens dociles et seulement préoccupés de croissance économique (Nussbaum 2011 : 35). Nussbaum assigne un certain nombre de buts à l'enseignement démocratique, buts dont elle pense qu'ils seront mieux atteints si on accorde une large place à l'enseignement des arts, de la raison et de l'imagination.

Elle énumère sept points qui lui semblent essentiels et que je vais résumer ici :

1. La capacité de raisonner sur des questions politiques ;

2. La capacité de reconnaitre ses concitoyens comme des égaux ;

3. La capacité de se préoccuper de la vie des autres ;

4. La capacité d'imaginer une variété de problèmes complexes «qui affectent l'histoire d'une vie humaine dans son déploiement ";

5. La capacité de juger avec un esprit critique ;

6. La capacité de penser au bien de son pays ;

7. La capacité de voir son pays comme une fraction d'un ordre mondial.

9 L'intérêt de la démonstration de Martha Nussbaum est qu'elle situe l'enseignement de la littérature et des arts dans une double perspective.

Elle propose tout d'abord une interprétation très personnelle de la place ou de la fonction de l'art dans nos sociétés. Très lié au jeu (elle cite abondamment Winnicott), l'art permet selon elle le développement de "l'imagination narrative », c'est-à-dire «la capacité à imaginer l'effet que cela fait d'être à la place d'un autre » (Nussbaum 2011 : 121). De cette capacité à créer de l'empathie ou de la sympathie, Nussbaum fait la clé de voute d'un enseignement démocratique : capacité à se décentrer et à partager des émotions, capacité à appréhender des cultures spécifiques. Jacques Bouveresse, lecteur de Nussbaum, défend à son tour l'idée que le roman est un " outil philosophique » supérieur dans la mesure où il dispose d'un «pouvoir d'éclaircissement plus grand de réalités énigmatiques ou obscures $^{7}$ ».

11 En plaidant pour une « culture de l'imagination », Bouveresse, comme Nussbaum, montre que, dans la sphère publique, y compris dans des contextes juridiques, les œuvres littéraires convoquent le possible à côté de l'habituel, et qu'elles nous somment de prendre en considération « les formes modifiables de la société (Martha Nussbaum) ${ }^{8}$ ».

12 Mais le plaidoyer de Martha Nussbaum en faveur d'une éducation aux arts ou par les arts s'inscrit dans un autre contexte. Sa référence constante aux grands pédagogues qui ont 
marqué l'histoire de l'enseignement (Pestalozzi, Froebel, Dewey, Tagore...) situe d'une certaine manière l'enseignement des humanités. Le rôle des arts à l'école est, pour elle, étroitement lié à la construction du sens critique, par le débat, l'échange socratique, qui permet seul de prendre la mesure de l'autre, de développer une écoute respectueuse et de se construire dans la relation à l'autre, à tous les autres. Les exemples qu'elle prend relèvent le plus souvent d'une pratique artistique et font peu de cas de la révérence due aux grands modèles : une pratique de la danse en atelier, un chœur d'enfant à Chicago, etc. Pas plus Nussbaum que Bouveresse ne prétendent s'inscrire dans une tradition de déférence aveugle aux œuvres du passé. Bouveresse rappelle au contraire, pour inciter à la vigilance, que «la relation que nous entretenons avec [la littérature] est restée fondamentalement religieuse et n'a jamais été réellement sécularisée "; il cite Bourdieu, qui assimilait à des «bavardages de sacristie » "les lieux communs bien pensants » de Danielle Sallenave.

\section{Le reliquaire}

Forcément, l'enseignement de la culture humaniste (et de l'histoire des arts) a quelque chose à voir avec ce que François Flahault appelle "Le culte des reliques ${ }^{9}$ »: "l'enchâssement de l'objet dans un discours ", «le culte des héros, saints ou artistes ». Forcément, l'enseignement de la culture humaniste, parce qu'il vise la construction d'une culture commune et la transmission de valeurs, fait courir à la culture le risque de «la figer en biens culturels et en leur détestable rationalisation philosophique, les prétendues valeurs culturelles ${ }^{10} »$.

14 Le socle commun de compétence, sans définir la culture humaniste, lui attribue des fonctions: elle contribue..., permet..., enrichit..., participe à... etc. Cette définition en creux, assortie de références dispersées à plusieurs champs disciplinaires qu'il convient de rapprocher pour construire les connaissances, capacités et attitudes correspondantes, laisse le champ libre à de multiples interprétations. Mais elle induit plutôt une pratique, un ensemble d'actions ou d'expérimentations alors que les premiers documents proposés aux enseignants pour donner une illustration de la matière histoire des arts se présentent davantage comme des listes de repères ou de monuments désignés à l'étude admirative ${ }^{11}$. Bref, entre pratique authentique des émotions (liées à l'expression artistique ou à la réception des œuvres) et déférence obligée à un panthéon de productions anciennes, l'enseignement de la culture humaniste semble condamné à hésiter.

Plus que tout autre enseignement en effet, celui de la culture humaniste pose la question des objets enseignés et enseignables, du rapport des apprenants à la connaissance attendue et, par conséquent, des discours qui se tiennent dans la classe ou en formation. À la fin d'un article brillant ${ }^{12}$, où il vient de montrer que contrairement à certaines affirmations simplificatrices, la pédagogie du français n'a jamais été une "pédagogie de l'expression », Éric Dumaitre, reprend à son compte les analyses de Bourdieu pour qui la culture se communique assez largement de façon implicite. Les «humanités» qui tiennent davantage de la posture ou de l'éthos (une certaine familiarité avec les œuvres et avec la langue) ne sont-elles pas plutôt lentement édifiées depuis la petite enfance, bien davantage hors de l'école qu'à l'école ? Quant à l'apprentissage laborieux que dispense habituellement l'école (listes de chefs d'œuvres aussi lointains qu'inaccessibles, références imposées et inexplicables, gloses sophistiquées autant qu'énigmatiques) permet-il jamais de "gouter aux joies de l'exercice assuré et délié des facultés 
intellectuelles ", alors même que l'évaluation repose justement sur l'appréciation de compétences telles que l'aisance, le brio, voire la « distance ». L'acquisition scolaire de la culture, affirme-t-il, passe par des étapes ingrates qui ne devront en aucun cas être apparentes au moment d'une évaluation, laquelle méprise plutôt ce qui peut paraitre scolaire ou laborieux au profit d'une sorte d'incorporation des savoirs, suffisamment naturalisés pour autoriser l'aisance d'une posture détachée.

En bref, l'enseignement de la culture humaniste s'il se dégage des pratiques créatrices, s'il ne donne pas l'occasion aux élèves de construire un pouvoir et une posture, - seul véritable enjeu de la culture humaniste : l'autonomie -, et s'il se réduit à l'inventaire irrationnel (pour les sujets que sont nos élèves ${ }^{13}$ ) d'œuvres présentées comme des repères, n'aura inventé qu'une nouvelle manière d'organiser le reliquaire glacé des connaissances, sans véritablement prendre en charge les émotions, en accentuant encore l'écart entre les élèves.

17 Qu'en est-il exactement des propositions des circonscriptions en matière de culture humaniste?

\section{Arborescences}

La (re)naissance de la culture humaniste à l'école passe tout d'abord par l'émergence de structures et d'acteurs nouveaux, de dispositifs administratifs qui permettent aux acteurs de se regrouper pour partager leur réflexion, faire part de propositions, initier des projets ou diffuser des outils. C'est ce que font les groupes départementaux.

19 Le groupe départemental «Culture humaniste » de la Drôme ${ }^{14}$ est par exemple constitué de trois inspecteurs, un professeur en IUFM (Histoire-Géographie), cinq conseillers pédagogiques (deux spécialisés, trois en circonscription), trois maitres-formateurs et sept enseignants de terrain). Il est difficile de dire à quel point le fonctionnement de ces groupes est réel : sur le net, certains d'entre eux, n'affichent aucune actualité depuis de nombreux mois, d'autres se limitent à des liens vers d'autres groupes, vers le site du ministère ou en direction de rares ressources locales. Ce qui est sans doute intéressant, c'est de voir comment les échelons intérieurs de l'institution réagissent à partir d'une commande ministérielle : d'une part en créant des groupes de travail supposés prendre en charge la formation, dotés d'un plan d'action, d'un calendrier et constitués d'équipe pluricatégorielles; d'autre part en affichant une réorganisation des disciplines avec un étiquetage nouveau. Ainsi plusieurs académies ${ }^{15}$ recrutent-elles dès 2010, un conseiller pédagogique «Culture Humaniste, Option Arts Visuels ». Les conseillers pédagogiques Arts Plastiques, puis Arts Visuels existent depuis longtemps.

20 La nouveauté réside dans la restructuration de l'existant : les Arts Visuels sont désormais une option au sein d'un ensemble plus vaste, la Culture Humaniste, dont l'apparition réorganise les disciplines existantes, redéfinies comme autant de composantes d'une nouvelle macrodiscipline. Cette restructuration conduit à de nouveaux appariements, assez variables d'un département à l'autre. Ainsi, le Groupe départemental 36 (Indre $\left.{ }^{16}\right)$ est un groupe Culture Humaniste - HIDA (pour Histoire des Arts) alors que le rapprochement de la culture humaniste avec l'histoire des arts n'est pas toujours aussi clairement revendiqué.

21 Une architecture exemplaire est ainsi visible sur le site de la Manche $^{17}$ dont la page Culture Humaniste ouvre des liens vers quatre autres pages, dont les intitulés présentent 
une sorte de programme : 1/ Éducation artistique et culturelle, 2/ De la structuration du temps à l'histoire, 3/ De la structuration de l'espace à la géographie et 4/ Instruction civique et morale. De ces quatre pages, à la date où nous écrivons, seule la première n'est pas en construction et offre à son tour un programme très riche de liens divers.

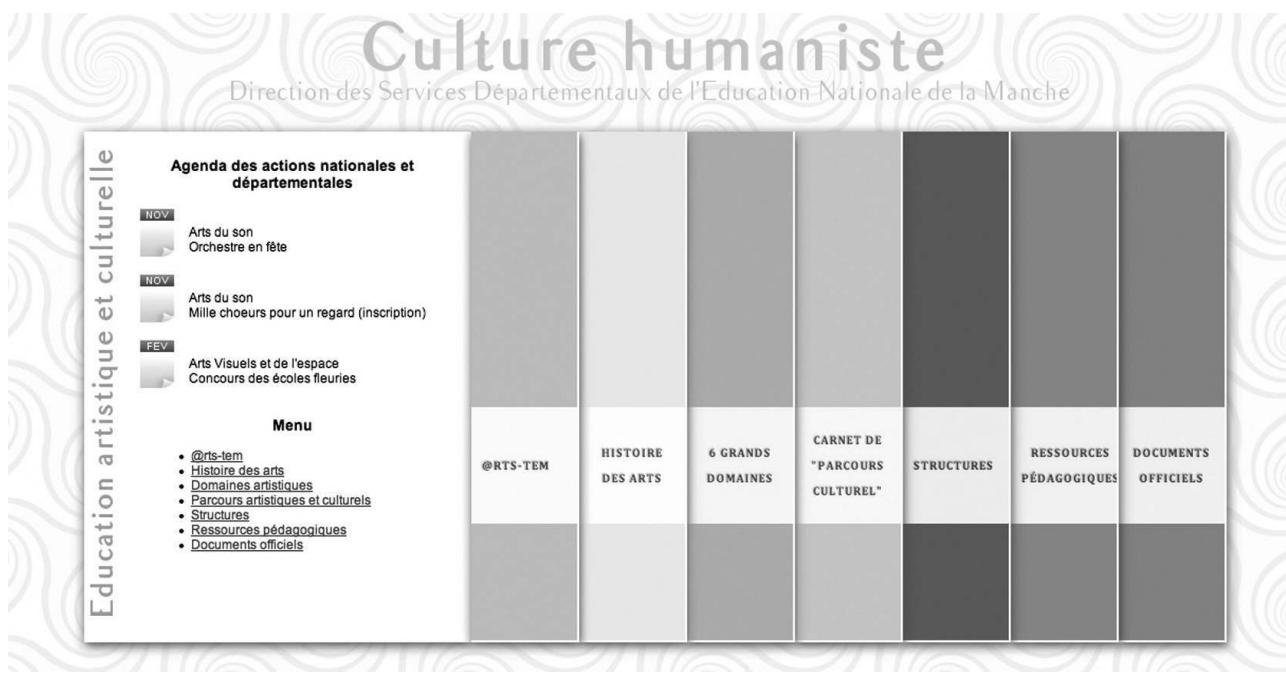

Le «menu » de l'éducation artistique et culturelle se subdivise ainsi une première fois de la façon suivante :

- @rts-tem propose des activités en relation avec l'histoire culturelle des cinq continents ;

- Histoire des arts ;

- Les six grands domaines artistiques ;

- Un carnet de parcours culturel ;

- Une carte interactive des structures du département ;

- Les ressources du CDDP de la Manche;

- Les textes officiels.

$\mathrm{Au}$ delà du formalisme toujours envisageable, la réorganisation des connaissances et des compétences induit un autre regard sur les anciennes disciplines, et appelle des pratiques nouvelles, même si celles-ci ne peuvent naitre instantanément. L'égalité des " domaines » accessibles à partir du troisième onglet du menu (les six grands domaines artistiques) établit par exemple un parallélisme explicite entre la littérature et les autres pratiques artistique: les arts du langage, au lieu d'être exclusivement associés à la maitrise de la langue, rapprochés des cinq autres grands domaines artistiques, devraient être perçus autrement par les enseignants comme par les élèves. 


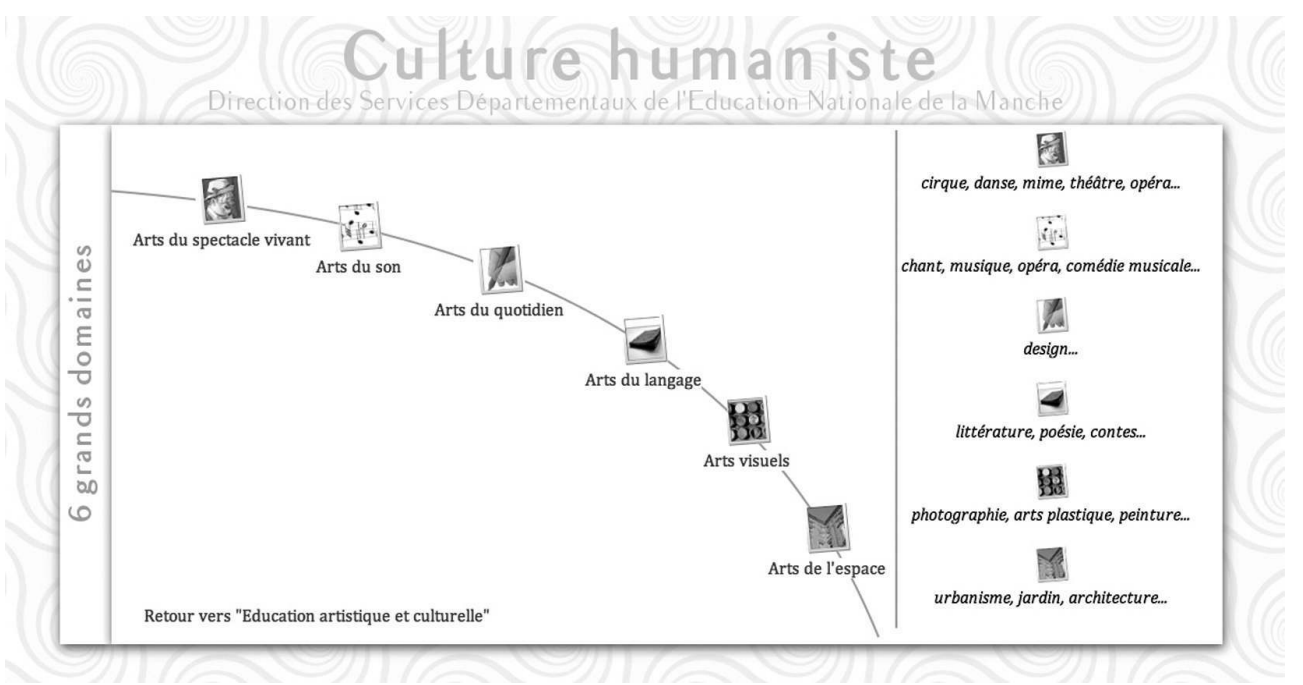

De la même façon, le théâtre, «art du spectacle vivant ", perd une forme de solidarité avec la littérature alors qu'il s'en trouve une autre avec le cirque : les Arts Visuels avaient déjà opéré une semblable révolution en intégrant la photographie ou le cinéma.

5 Ce souci d'architecture apparait encore dans les nombreux documents vierges mis à la disposition des enseignants : faute d'indiquer une activité ou une séquence réalisée ou réalisable, ils suggèrent un cadre, inscrivent les situations de classe dans une perspective pluridisciplinaire comme dans le document proposé à Alençon ${ }^{18}$. Le projet de "parcours culturel » de la classe se présente sous une frise qui rappelle les six «domaines» de création artistique et se décline en: Intitulé du projet et Domaine(s) artistique(s) concerné(s) ; Autres domaines ou disciplines concernés (histoire, géographie, sciences...) ; Rapport direct à des œuvres à des monuments à des objets; Artiste, Médiateur, Intervenant extérieur ; Structure culturelle ; Dispositif, Opération nationale ; Réalisations élèves et Communication finale. La formation passe par l'imposition d'une forme : l'action des enseignants inscrite dans un nouveau format suppose (sans les imposer) des correspondances ou des liens entre les "domaines ", entre des œuvres et des productions de classe, entre le cadre scolaire et des structures ou intervenants extérieurs.

Le souci d'articuler réception et production artistique apparait très nettement encore dans une autre fiche élaborée par l'inspection du Morbihan ${ }^{19}$. Les différents domaines de l'histoire des arts sont inclus dans une grille de programmation sur trois colonnes. La première répertorie différentes déclinaisons propres au domaine : pour les arts visuels, peinture, vidéo, installation... etc. La deuxième laissée provisoirement vierge doit permettre à l'enseignant de noter les situations qui ont permis de voir / écouter / rencontrer des œuvres; la troisième concerne les productions. Et la grille est répétée pour chaque cycle.

Mais que reste-t-il de cette redistribution des contenus disciplinaires et de la nouvelle architecture dans les modèles proposés ? Dans quelle mesure la culture humaniste offret-elle l'occasion de penser l'enseignement de façon pluridisciplinaire et de cultiver à côté de savoirs spécifiques un ethos particulier, un rapport personnel, sensible et autonome à la création, aussi bien comme récepteur que comme producteur? 


\section{Où le bât blesse...}

du mois permet de « développer l'ouverture culturelle des élèves et de soutenir une séquence de travail en arts visuels ». L'image est située dans l'œuvre et la biographie de l'artiste, son époque. Puis des activités ou des exercices à partir de l'œuvre ou directement sur celle-ci (dévoilement progressif, expérimentation de procédés, utilisation d'outils qui permettent aussi de « faire »). Les indications historiques semblent réservées à l'enseignant et ne doivent pas nécessairement être utilisées dans la séquence en classe (j'ai pris pour appui la fiche consacrée à Impressions Soleil Levant mais les fiches sont toutes construites selon ce procédé comme en témoigne le descriptif de la séquence liée aux fresques de Tarquinia : « Séance 1: Expérimenter un procédé pictural : "la couleur 
diluée", Séance 2: Découvrir l'image d'ancrage. Séance 3: Réaliser une composition décorative. Séance 4 : Confronter le travail vécu avec les satellites »).

Ainsi, par rapport aux questions que je posais un peu plus haut (quelle interdisciplinarité la culture humaniste favorise-t-elle ? Quel équilibre peut-on trouver entre problématique patrimoniale et approche sensible de la création artistique ?), les fiches consacrées à l'image $d u$ mois proposent des démarches très intéressantes de mise en relation $d u$ tâtonnement ou de l'exploration de matériaux et de la lecture personnelle d'images artistiques, mais l'architecture d'ensemble n'est qu'une enveloppe qui a permis de situer les arts visuels dans un répertoire d'actions ou de compétences qui ne sont pas davantage reliées entre elles.

Occupons-nous d'un autre exemple : L'académie de Nantes $^{23}$, circonscription de Montaigu, présente dix chapitres de ressources. Le cinquième, consacré à la culture humaniste et à l'histoire des arts comporte seulement trois documents :

1. Une progression pour le cycle 3 ;

2. Un lien vers un site consacré à la musique : les couleurs de l'orchestre ;

3. Un dossier (apparemment inspiré par une série d'animations pédagogiques) sur l'espace de l'école maternelle.

Plusieurs remarques sur cette page qui n’a pas été alimentée depuis 2011 :

- Le petit nombre de propositions témoigne de la volonté de "prendre place », d'ouvrir une rubrique qu'il a été impossible de compléter par la suite, et donc de la difficulté à inscrire dans le temps une prescription qui risque d'être perçue par les enseignants comme une marotte institutionnelle passagère ;

- L'hétérogénéité des trois documents manifeste une réelle richesse et d'autres difficultés : le très pertinent dossier sur l'espace en maternelle aurait très bien pu figurer ailleurs, le lien vers les instruments de l'orchestre est strictement disciplinaire, la très intéressante progression en Histoire est en revanche très symptomatique du rôle attribué à la discipline historique. La vie quotidienne, les grands évènements, l'évolution du pouvoir et de la religion : ces trois rubriques permettent de mettre en relation des faits historiques, et des pratiques socioculturelles ou artistiques. En clair, presque tout ce qu'on peut faire à l'école relève effectivement de la culture humaniste, chaque discipline artistique se suffit à ellemême pour l'incarner, mais l'histoire est l'instrument privilégié pour aborder de façon un peu convergente des questions d'ordre culturel.

- Une situation d'écriture, sur le même document, permet d'assurer des passages entre savoirs historiques et pratique expressive : « Un petit Parisien écrit à un petit chouan ou Vendéen. (mise en écrit descriptif à partir des tableaux d'intérieur d'époque, à partir d'évènements explicites comme la prise de la Bastille ou les guerres de Vendée) ».

Cette intéressante proposition de "décloisonnement» est ici ponctuelle dans un dispositif qui, une fois de plus s'attache principalement à l'histoire. La culture humaniste se traduit en effet le plus souvent par des propositions de progression en histoire ou par des scénarios pédagogiques centrés sur l'étude de l'Histoire (les cent ans de la guerre de 14). Pour décloisonner l'enseignement des arts visuels et de l'éducation musicale, le site $\mathrm{du}$ Morbihan ${ }^{24}$ propose une démarche ambitieuse, puisque non content de déterminer la place de ces disciplines dans le contexte du pilier 5, les auteurs s'efforcent de décliner pour chaque « pilier » des types d'action compatibles ou complémentaires. Ainsi s'investir dans un projet choral apparait à la fois compatible avec le pilier 5 (« Projets musicaux mis en relation avec les enseignements - progression, évaluation - »), et avec les piliers 6 et 7 
(compétences sociales et civiques, autonomie et esprit d'initiative). Chacune de ces activités, loin de s'éloigner des préoccupations propres à la discipline se justifie d'un ancrage dans un contexte et plus large.

Un document publié sur le site de la circonscription de Saint Gilles Croix-de-vie ${ }^{25}$ pourrait illustrer un autre péril, propre à la pluridisciplinarité cette fois-ci. Intitulé, Littérature de jeunesse et culture humaniste, le document propose une bibliographie classée d'ouvrages qui fournissent "une entrée intéressante pour aborder les différents domaines de la culture humaniste ». Les différentes rubriques sont éloquentes ainsi que les commentaires associés ${ }^{26}$ : la littérature est un instrument de connaissance du monde, et nul doute que c'est une des fonctions des livres d'ouvrir à ses lecteurs des espaces ou des mondes différents. On peut cependant regretter que la spécificité des études littéraires (et non pas des textes littéraires) ne soit pas aussi convoquée comme une des voies d'accès à la culture humaniste: intelligence critique, habileté interprétative, partage social des émotions. Autrement dit, la démarche qui consiste à solliciter une discipline pour entrer dans la culture humaniste, l'en exclut de fait : si elle en constitue le seuil, elle ne peut en être une composante !

\section{Quelles conclusions tirer de cette brève exploration du web?}

1/ Que la culture humaniste à l'école est avant tout un classement, une façon d'organiser les apprentissages, dont les différentes présentations disponibles sur le net permettent d'illustrer à la fois le caractère volontariste, et les résistances qu'elle occasionne ${ }^{27}$; de ce point de vue, l'appel à la culture humaniste ouvre une double sollicitation: l'une patrimoniale, attentive à la transmission d'un héritage, l'autre soucieuse de restaurer un rapport au monde, fait de curiosité et d'ouverture passionnée aux savoirs et aux arts. La globalité structurée que suggère cette nouvelle (mais très ancienne) présentation des apprentissages se heurte au découpage par discipline comme aux commodités de la mise en œuvre des séances. D'où cette tension et cet écart entre les propositions qui restent sur un découpage pratique et celles qui cherchent à imposer des correspondances, une synergie, une appréhension globale du temps et de l'espace pédagogique.

41 2/ Que l'histoire est sans doute, par le biais de l'Histoire des arts, la discipline centrale qui permet le plus aisément de fédérer des connaissances multiples ${ }^{28}$; que le récit historique est sans doute le discours qui permet le plus facilement de donner une forme à cette globalité, d'où l'implication des spécialistes d'histoire dans ces dispositifs, d'où la limitation parfois à l'ouverture sur le monde (géographie, histoire) de certaines propositions, aux dépens d'une attention plus particulière aux expressions de ce rapport au monde dans les arts, qu'ils s'appuient sur le verbal ou sur le non verbal.

3/ Que les nombreux répertoires numériques offerts à l'attention des enseignants fournissent des ressources incomparables, dont il faudrait se servir autrement que pour illustrer des cours d'histoire (ou pour élaborer le reliquaire de plaisirs éteints), pour conduire à la curiosité, au sens critique et à l'autonomie de jugement constitutifs d'une culture humaniste. À côté de cela, la mise en projet est une vieille lune de la pédagogie, toujours et heureusement, bien vivante: les projets d'école, dont le net fournit de nombreux exemples, ou de classe permettent aux élèves d'observer, d'analyser ou de penser et de créer, dans ou à partir des œuvres du passé, en les (r)éveillant. Sans 
référence systématique à la culture humaniste, sans s'ancrer dans l'une de ces architectures sophistiquées qui situent les apprentissages les uns par rapport aux autres, les projets d'école témoignent de cette volonté des enseignants de faire construire ensemble des objets, des relations entre les lieux, les époques, les façons de faire ou de voir.

Je terminerai donc sur un dispositif exemplaire, le projet "Images et patrimoine" de la circonscription d'Albi ${ }^{29}$ : en mobilisant géographie, histoire, histoire des arts, littérature et production d'écrits, en invitant à mieux regarder et comprendre le proche environnement des élèves, en s'inscrivant sur la toile avec les images et les textes d'élèves de tous les horizons, il offre une belle illustration de l'humanisme, normalement et quotidiennement, à l'œuvre chez nos collègues.

\section{NOTES}

1. La connaissance de l'écrivain, Agone, 2008.

2. Charles Dickens, Temps difficiles, in Dombey et Fils, trad. Andhrée Vaillant, La Pléiade, Gallimard, 1956

3. «Fille numéro vingt, dit le Monsieur, souriant avec la force tranquille de la science » (p.1010)

4. id., p. 1012

5. Martha Nussbaum, Love's knowledge. Essays on philosophy and literature, Oxford, Oxford UP, 1990.

6. Martha Nussbaum, Les émotions démocratiques, Comment former le citoyen du XXIe siècle, Climats, 2011.

7. id., p.19

8. id., 155-161.

9. Flahault François (1997), «L'artiste créateur et le culte des restes, in Communications 64, La création, p.15-53, pp. 19 et sqq.

10. Adorno, Prismes, 10-22

11. Qu'on se réfère à la mise en garde de Claude Lelièvre sur la sacralisation qu'induit une culture commune: «On pense en effet qu'il convient de ne mettre sous les yeux des élèves que les plus belles pages, les plus morales, à la fois modèles d'éloquence et modèles de vertu. Des «morceaux de bravoure ", en somme. D'où une pédagogie du «modèle ». D'où une éducation où l'on se soucie en premier lieu des «bonnes fréquentations » (il s'agit de l'élite, et de l'élite sociale, ne l'oublions pas). D'où, aussi, des « dérives ». Si l'on en juge par une enquête TNS-Sofres qui a été menée en 2003, l'enseignement de la langue française et de la littérature est l'un de ceux qui suscitent le moins « l'envie de découvrir et d'apprendre ». Chez les élèves de onze à dix-huit ans, il arrive en dixième position, à bonne distance non seulement du sport et de la musique, mais aussi des nouvelles technologies ou de l'histoire et de la géographie. (Le français aujourd'hui $n^{\circ}$ 167, 2009, p.11-17: Culture humaniste et "socle» de l'école obligatoire (tout le reste est littérature).

12. Je m'appuie ci-dessous en grande partie sur les arguments d'Eric Dumaitre dans cet article (2011) : Quelques variantes d'un lieu commun pédagogique : la formation de l'esprit par l'étude des lettres, Spirale 47, La Culture de l'expression, p. 97-121. 
13. L'apport de la didactique du français et les nombreuses contributions sur le lecteur ou le scripteur comme sujets devraient nous mettre à l'abri de ce genre de dérive. Mais les choses sont toujours plus compliquées dans les classes que dans les essais de didactique ou les textes prescripteurs.

14. http://www.ac-grenoble.fr/iennyons/neosite/spip.php?article928 (site consulté le 5 septembre 2013).

15. Ce n'est pas le cas de l'Académie de Montpellier qui recrute un conseiller pédagogique généraliste en «Culture Humaniste »: http://www.ac-montpellier.fr/doc_ia34/mouvement/ poste_profil/CPD_CULT_HUM.pdf

16. L'éducation artistique http://www.ac-orleans-tours.fr/dsden36/circ_le_blanc/pedagogie/ culture_humaniste_hida/

17. http://www.ac-caen.fr/ia50/circo/actionculturelle/arts.html\#

18. http://www.ac-caen.fr/ia61/circos/alencon/blog/index.php?category/ACTION-CULTURELLE

19. http://www.ia22.ac-rennes.fr/jahia/webdav/site/ia22/shared/Education\%20artistique\%20et \%20culturelle/schema\%20departemental/06DLE,2007\%2012\%2020\%20pratiques\%20cultrelles.pdf 20. op. cit.

21. L'université Bordeaux 3 ouvre en 2012 une Licence pluridisciplinaire Culture humaniste et scientifique, mais les sites consultés n'offrent que très occasionnellement des correspondances entre la culture humaniste et la culture scientifique : les liens privilégiés concernent plutôt l'histoire des arts.

22. La Haute-Savoie met en ligne un annuaire classé des ressources locales en matière d'intervenants extérieurs. (http://www.ac-grenoble.fr/arts-culture-humaniste74/spip.php? rubrique4). Le site du CNDP offre, bien entendu, un répertoire tout à fait remarquable de ressources : http://www.cndp.fr/crdp-paris/Ressources-sur-la-culture

23. https://www.google.com/search?q=par\%C3\%A9+culture

+humaniste\&rlz=1C5CHFA_enNC507NC507\&oq=par\%C3\%A9+culture

thumaniste\&aqs=chrome..69i57.6586j0\&sourceid=chrome\&ie=UTF-8\#q=vincent+par\%C3\%A9

+culture+humaniste\&safe $=$ off

24. http://www.ia22.ac-rennes.fr/jahia/webdav/site/ia22/shared/Education\%20artistique\%20et \%20culturelle/schema\%20departemental/01_DLE\%20les\%20enseignements\%20artistiques\%

20dans\%20le\%20socle\%20commun.\%E2\%80\%A6.pdf

25. http://cic-stgilles-ia85.ac-nantes.fr/spip.php?article236

26. «Passé proche: Découvrir au fil d'albums comment vivaient nos parents, nos grandsparents. Comprendre l'évolution des sociétés. Apprendre la tolérance. Contes, théâtre et poésie : Poésies et contes de chez nous et d'ailleurs à découvrir, à savourer, à comparer. Calligraphie, écritures et langues différentes... Carnets de voyage : Comment raconter, illustrer, mettre en page ses voyages. Dessins ou photos, noir et blanc ou couleur, impressions ou textes informatifs... découvrir des peuples lointains ou voyager dans sa tête. Grands voyages : Réels ou fictifs, des récits de grands explorateurs. Récits de vie à une autre époque : Permet aux élèves de découvrir la vie à d'autres époques à partir des aventures d'un enfant de leur âge. La guerre : Pour aborder les guerres de 14-18, de 39-45 ou d'Algérie ou la montée du totalitarisme. Récits de vie d'ici et d'ailleurs : Pour découvrir la vie et les coutumes tout autour de la planète. (id.)

27. La formation initiale n'est pas plus à l'aise sur cette question. Ainsi, un seul ouvrage de préparation au concours porte le titre Français et culture humaniste. Les auteurs, toutes deux agrégées de Lettres, ont choisi un plan qui privilégie la méthodologie de l'épreuve (100 pages avec des exemples développés), puis les questions de langue (107 pages) puis la présentation juxtaposée de l'histoire et de l'épistémologie des disciplines scolaires regroupées sous cette étiquette. Chacun de ces domaines est étudié successivement et à aucun moment le «Panorama des problématiques» disciplinaires ne s'efforce de bâtir des liens entre celle-ci ou, tout au moins de les comparer explicitement. Paru deux ans plus tard, chez le même éditeur, le 
livre de Manuelle Duszynski porte un titre voisin mais, en collant sans doute de plus près à la réalité de l'épreuve rappelle les programmes de français et d'histoire, limite la présentation des disciplines artistiques au seul volet de l'Histoire des arts. Foucher (2013) distingue nettement une partie Histoire et une partie Français. Les autres ouvrages de préparation à l'épreuve de Français du CRPE (Dunod 2012 ; Hatier 2010) privilégient la méthodologie de l'épreuve et la connaissance de la langue. Seul, l'ouvrage de Jeanne-Antide Huynh, Patricia Monti-Lovichi et Virginie Brincker (Réussir le Français à l'écrit, Atlande 2012.) consacre deux chapitres de dimension non négligeable au statut culturel de la littérature dans la production écrite (Culture des textes/ textes et culture(s), partie consacrée d'une part aux genres littéraires, à la littérature pour la jeunesse, aux littératures francophones et aux rapports entre lecture et lecture littéraire ou écriture et écriture littéraire), aux arts et à la représentation du monde (brève histoire des arts du moyen-âge à nos jours).

28. Qu'on consulte sur le site de l'académie de Versailles, les très belles frises enrichies de multiples liens qui permettent de naviguer véritablement entre des pôles multiples: http:// www.ac-versailles.fr/public/jcms/p1_179049/frise-numerique-histoire-et-histoire-des-arts.

29. http://pedagogie.ac-toulouse.fr/primaide/crtice81/sites81/display.php? idss $=102 \&$ selectrub $=39$

\section{RÉSUMÉS}

L'enjeu de l'article est de proposer une réflexion sur les perspectives ouvertes par l'introduction de la culture humaniste à l'école. Il s'agit d'analyser, à travers les propositions de différents sites académiques, les évolutions récentes de l'enseignement des humanités dans le système éducatif. Ces documents de formation témoignent de la façon de répondre à une commande institutionnelle, ils manifestent une forme de volontarisme en soulignant les liens entre les différentes disciplines qu'ils s'efforcent de reconfigurer, tout en indiquant les difficultés ou les résistances.

The aim of the paper is to propose a reflection on the opportunities created by the introduction of humanistic culture as a part of schools' curricula. The analysis concerns recent developments in the teaching of humanities, through the offerings of different academic websites. These training materials and tools provide direct insight into how actors on the ground respond to an institutional policy; they indicate a form of proactive approach by developing links between different disciplines that they try to reshape, while indicating difficulties or resistances.

\section{INDEX}

Mots-clés : commande institutionnelle, enseignement des humanités, supports de formation

Keywords : humanistic culture curricula, institutional policy, training materials 
AUTEUR

FRANÇOIS QUET

Université Lyon 1, Traverses 19-21 